\title{
Intra-operative Real-Time 3-D Information Display System Based on Integral Videography
}

\author{
Hongen Liao ${ }^{1}$, Susumu Nakajima ${ }^{2}$, Makoto Iwahara ${ }^{3}$, Etsuko Kobayashi ${ }^{3}$, \\ Ichiro Sakuma ${ }^{3}$, Naoki Yahagi ${ }^{3}$, Takeyoshi Dohi ${ }^{3}$ \\ ${ }^{1}$ Department of Precision Machinery Engineering, Graduate School of Engineering \\ ${ }^{2}$ Department of Orthopedic Surgery, Graduate School of Medicine \\ ${ }^{3}$ Institute of Environment Studies, Graduate School of Frontier Sciences \\ The University of Tokyo, 7-3-1 Hongo, Bunkyo-Ku, Tokyo 113, Japan \\ \{liao, susumu, iwahara, etsuko, sakuma, yahagi, dohi\}@miki.pe.u-tokyo.ac.jp
}

\begin{abstract}
A real-time 3-D surgical navigation system that superimposes the real, intuitive 3-D image for medical diagnosis and operation was developed in this paper. This system creates 3-D image based on the principle of integral photography (IP), named "Integral Videography (IV)", which can display geometrically accurate 3-D image and reproduce motion parallax without any need of special devices. 3-D image was superimposed on the surgical fields in the patient via a half-silvered mirror as if they could be seen through the body. In addition, a real-time IV algorithm for calculating the 3-D image of surgical instruments was used for registration between the location of surgical instruments and the organ during the operation. The experimental results of puncturing a point location and avoiding critical area showed the errors of this navigation system were in the range of $2 \sim 3 \mathrm{~mm}$. By introducing a display device with higher pixel density, accuracy of the system can be improved.
\end{abstract}

\section{Introduction}

With the advancements of medical imaging and computer technology, the effective use of diagnostic images and image-guided surgical navigations have been a focus of discussion. They usually show 3-D information in pre-operation images to surgeons, as a set of 2-D sectional images displayed away from the surgical area. Surgeons have to follow some extra procedures to perceive 3-D information of the patient during surgery. Firstly, they need to turn their gaze to the computer display showing the navigation images. Next, they must reconstruct the 3-D information in their minds, with the help of experience and anatomical knowledge. Lastly, they must turn their eyes back to the surgical area and register the 3-D information reconstructed in the minds with the actual patient's body. These procedures interrupt the flow of surgery and the reconstructed 3-D information sometimes differs between individual surgeons. To solve these problems, medical images should be displayed threedimensionally in the space that coincides with patient's body.

To superimpose images over the observer's direct view of object, a lot of computer display techniques were presented. One of them is image overlay, which is a form of "Augmented Reality" in that it merges computer-generated information with real world images. Blackwell [1] used binoculars stereoscopic vision display system to show 3-D images with this method. This display method reproduces the depth of 
projected objects by the fixed binoculars disparity of the images. However, this does not always give the observer a sense of depth and the motion parallax cannot be reproduced without wearing a tracking device. As well as limiting the number of observers, this also causes visual fatigue. Consequently, systems based on binoculars stereoscopic vision are not suitable for surgical display.

In this study, we used 3-D image named Integral Photography (IP), which was first proposed by M.G.Lippmann[2] in 1908. IP records and reproduces 3-D image using a "fly's eye" lens array and photographic film. Masutani et al. recorded an IP of medical 3-D objects on the film and applied it to clinical diagnosis and surgical planning [3]. Igarashi et al. proposed a computer-generated IP method that constructs an image by transforming the 3-D image about the object into 2-D coordinates on the computer display, instead of taking a photograph [4]. However, the computing time for creating IP was so costly that the system could not be practical for rendering medical objects.

To overcome these limitations, we devised a new rendering algorithm for computer- generated IP and developed a practical 3-D display system [5] and applied it to a clinical field of orthopedic surgery [6]. Moreover, in the clinical application, the 3-D image of surgical instrument inserted into the organ also should be shown its realtime location. Consequently, a real-time rendering algorithm for intra- operative image information should be developed, especially for displaying the accuracy location of the intra-operative instrument.

In this paper, we present a real-time 3-D surgical navigation system that superimposes the real, intuitive 3-D image for medical diagnosis and operation. This system creates 3-D image based on the principle of integral photography, named "Integral Videography (IV)", which can be updated following the changes in operator's field of vision. 3-D image was superimposed on the surgical fields in the patient via a half-silvered mirror as if they could be seen through the body. In addition, a real-time Integral Videography algorithm for calculating the 3-D image of surgical instruments was used for registration between the location of surgical instruments and the organ during the operation. The accuracy of the navigation system was evaluated through in vitro experiments with phantoms.

\section{Method}

\subsection{System Configuration}

The 3-D real-time surgical navigation system developed in this study consists of the following components:

- Position tracking system POLARIS ${ }^{\mathrm{TM}}$ Optical Tracking System, Northern Digital Inc., Ontario, Canada

- 3-D data source collection equipment (such as MRI, CT)

- IP display (consist of LCD with XGA, lens array, half-silvered mirror, supporting stage)

- $\quad$ Personal computer Pentium 800MHz Dual CPU

The source image on the IP display is generated from the 3-D data by the algorithm described later in this paper. Any kind of 3-D data source, such as magnetic resonance (MRI) or Computerized tomography (CT) images can be processed. 


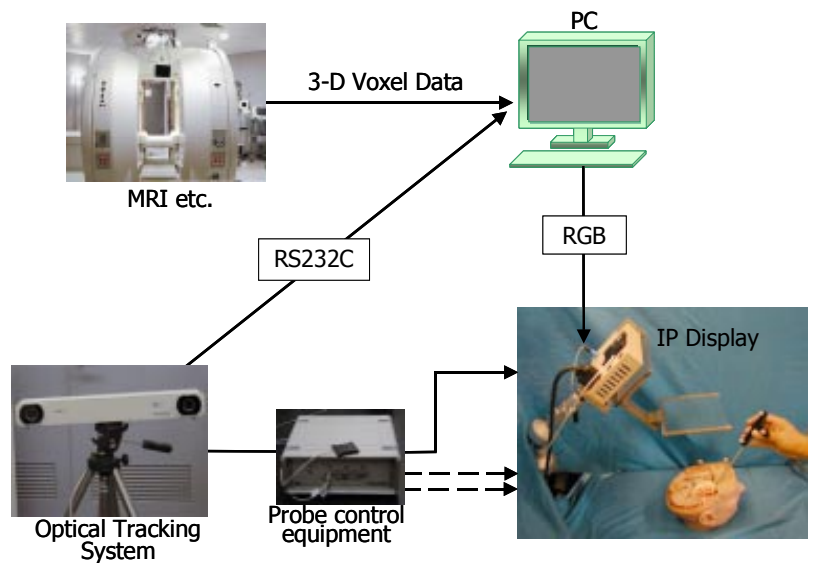

Fig.1 System configuration

We use optical 3-D tracking system to track the position of surgical instrument and organ during the operation. The position and orientation of IP display can be also measured for image registration. With these data, we calculate the relative position of surgical instrument to organ. Both of the patient organ model/target and surgical instrument are represented in the form of IP. The resultant IPs are displayed in realtime on IP display.

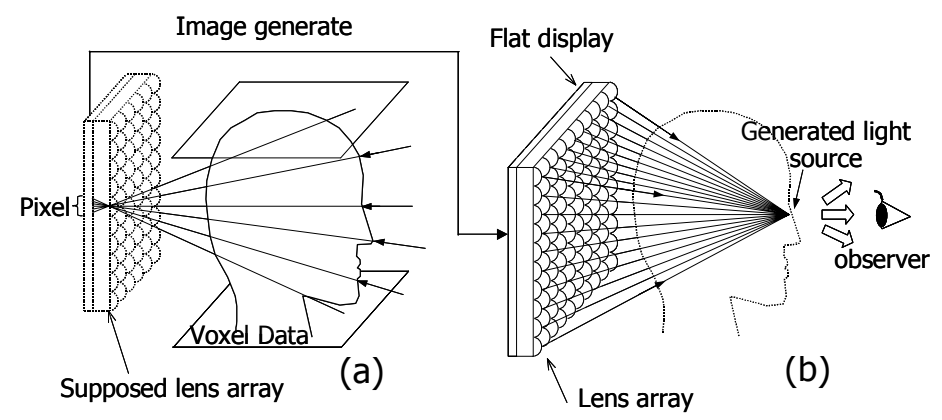

Fig.2 Principle of Integral Videography. The figure shows how to generate and reproduce a 3$\mathrm{D}$ object by IV. Light rays reflected at the first point seen from the observer's side on the 3-D object pass through the centers of all lenses on the array and are redisplayed on the flat display. When the image was lit from behind, light rays intersect at the original point to become a new light point.

In the proposed computer-generated IP, each point showed in a 3-D space is reconstructed by the convergence of rays from pixels on the computer display, through lenses in the array. The observer can see any point in the display from various directions as if it were fixed in 3-D space. Each point appears as a new light source (Fig2.b), and then a 3-D object can thus be constructed as an assembly of such reconstructed light sources. Coordinates of those points in the 3-D object that 
correspond to each pixel of the flat display (for example: LCD) must be calculated for each pixel on a display. Fig.2a Shows 3-D IP image's rendering method that calculates coordinates of one point for each pixel of the flat display.

The fundamental layouts of this system include the operator, 3-D image on IP display, half-silvered mirror, reflected image, patient in this system, the relations of them are shown in Fig.3.

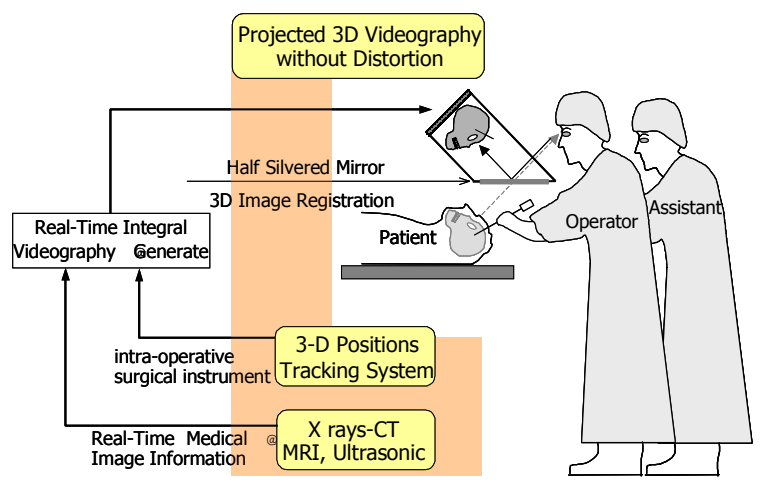

Fig.3 Fundamental layout for surgical registration

\subsection{Generate a Real-Time IP for Organ and Surgical Instrument}

(1) Method of generating IP for organ and surgical instrument

We can create a clear and detailed stationary IP image by using the fundamental method shown in Fig.2. However, the calculating time of this method for searching the first point seen by observer was too costly to be used as a real-time IP creating. Moreover, the intra-operative situation of the surgical instrument change frequently compared to the organ. It is very difficult to create a real-time intra-operative IP when the surgical instrument is calculated together with the organ. Consequently, we present a new method that creates organ and surgical instrument's IP respectively firstly (Fig.4), and then combine them together. One of integrated IP image of brain and inserted surgical instrument creating with this method is shown in Fig.5.

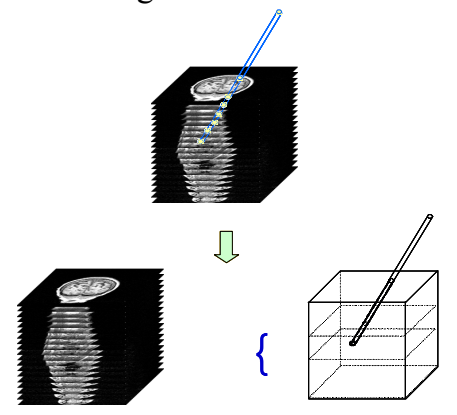

Fig.4 Method for dealing with organ and surgical instrument for making real-time IP

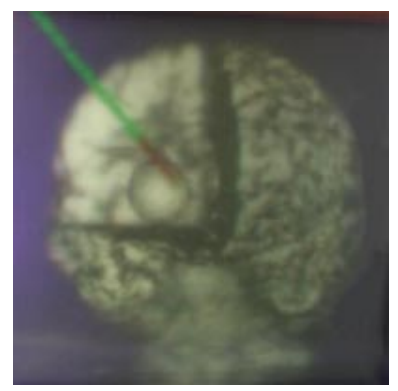

Fig.5 A cross section and the foci of brain IP 
(2) Fast IP rendering algorithm for 3-D object and surgical instrument

Although the fundamental method for IV can achieve all of the image information without any escape, the rendering time for the entire pixel on the flat display is so costly that cannot be used for real-time IP creating. We develop a special rendering method for medical IP, named jump-rendering method (Fig.6). It search the image voxel data every $2^{n} n=3$ or more pixels on the searching line. When the searched position (point on the searching line like A) enter the area of organ, the searching point will return back to the last step, and then re-research the pixel data every one pixel. In addition, we only calculate the IP in the necessary area of lens array (Fig.7). The calculation time with this method will be shorted to below 1 seconds, which is satisfied with the requirements of real-time IP.

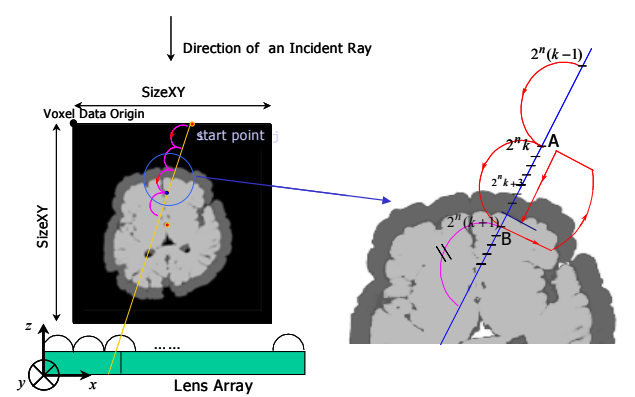

Fig.6 Fast rendering algorithm for IP of organ

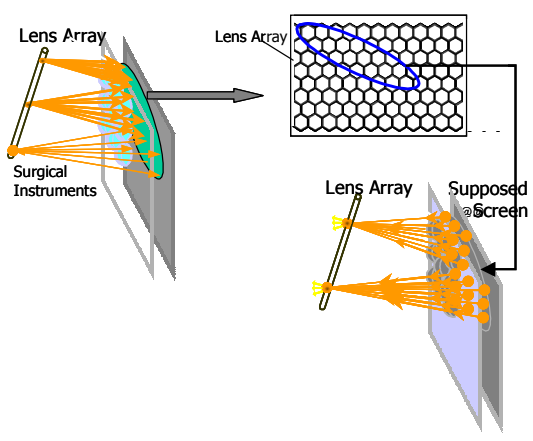

Fig.7 Real-time IP rendering algorithm for surgical instrument

\subsection{Registration Method}

Because the relationship between the location of the projected 3-D image and that of the IP display can be found from calculation, registration between the 3-D image and the patient's body could be performed by registration between the IP display and the patient's body. The relation between the surgical instrument and the organ can be also confirmed by using optical tracking probes. In this study, we use optical tracking probe (Fig.8) to measure the intra-operative situation of IP display, and then we can achieve the display coordinate $\Sigma$ dis form the transform of $\Sigma$ dis $-\Sigma s t d$. The organ and surgical instrument coordinate $\Sigma$ org can also be achieved as the same way. With the registration of $\Sigma d i s$ and $\Sigma o r g$, we can combine the 3-D image of intra-operative surgical instrument and organ with the patient's body (Fig.8). Once the displayed objects have all been transformed into the same coordinate system via this kind of calibration and registration transformations, they will be appeared to the operator's eyes exactly as if they existed in their virtual spatial locations. 


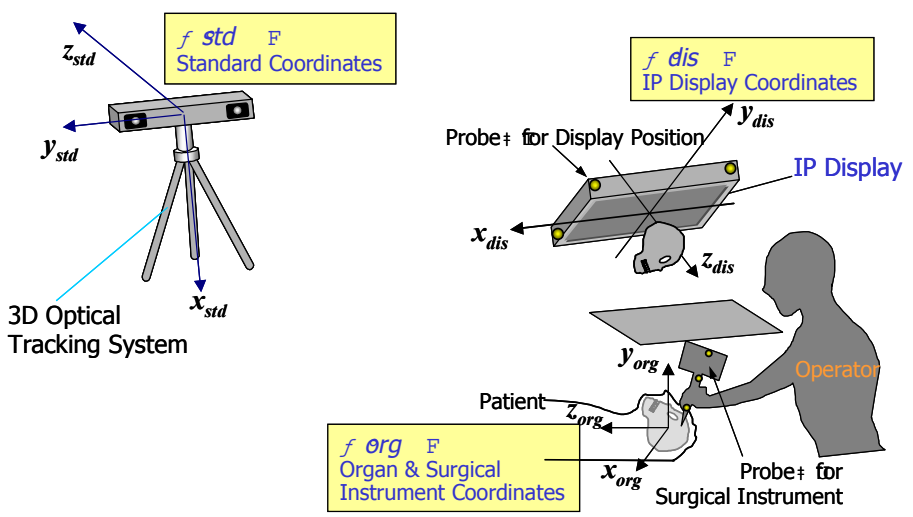

Fig. 8 Transformations between coordinate systems

\section{Experiments}

\subsection{Experimental Equipment}

Using the registration method shown in 2.3, we can coincides the position of the 3-D image theoretically with that of objects. However, some errors in the recognized positions of the points in the image are inevitable. So we measured the accuracy of the recognized location of the registered image to the real object by using a $80 \mathrm{~mm}$ cubic phantom objects, which include four MRI markers (a column capsule filled with oil, diameter of about $7 \mathrm{~mm}$ ) on its surface and a ball (diameter $15 \mathrm{~mm}$, material in clay, regarded as target in the puncturing experiment), two poles (diameter $2-4 \mathrm{~mm}$, regarded as vessel in the avoiding experiment) inside (Fig.9).

The 3-D image generated from MRI was superimposed on the phantom with the registration method mentioned above. The position corresponding to the markers were displayed in the 3-D image and their coordinates were measured three times by the optical tracking system (Fig.10). The mean value of differences between the measured and the true coordinates of four markers $M_{i}(i=1 \sim 4)$ is $1.13 \mathrm{~mm}$ and the S.D is $0.79 \mathrm{~mm}$.

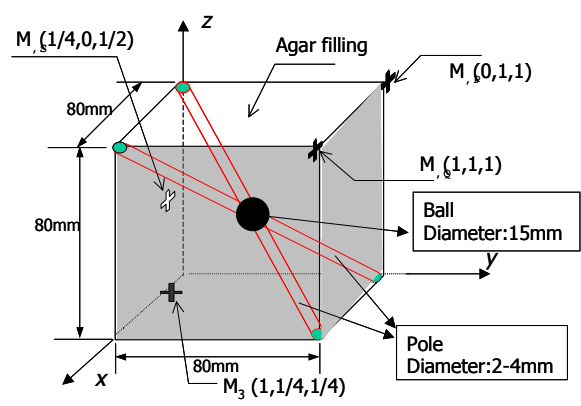

Fig.9 Scheme of the cubic phantom
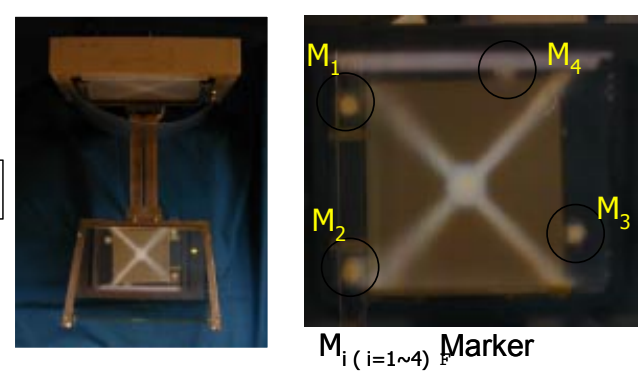

Fig. 10 Registration result with IP display 


\subsection{Real-Time Puncture and Avoidance Experiment}

- Real-time puncture experiment

We measure the spatial location of surgical instrument (a small needle) by tracking the probe fixed on surgical instrument with an optical tracking system. The tip positions and inserted point of the needle can be calculated. These position data will be used for creating an intra-operative IP image of surgical instrument. Fig.11 show an instantaneous situation of the inserted part of surgical instrument when the tip point of it

(a)

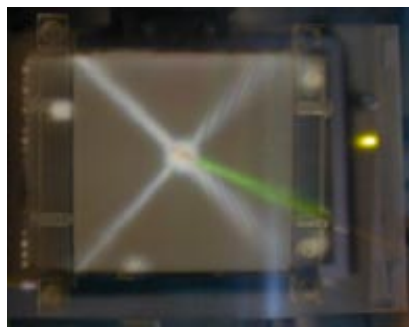

(b)

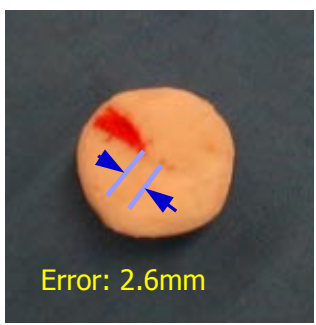

Fig.11 (a): An instantaneous situation of IP image when the tip point of surgical instrument arriving at target. (b): The result of puncture experimentation.

arriving at target in phantom. After finishing puncturing experiment, in order to examine the accuracy, we withdrew the surgical instrument out of phantom and injected colored-ink into the traces of puncture from the entrance point of the needle. The puncture error was $2.6 \mathrm{~mm}$ by measuring the traces remained in the clay, as shown in Fig.11 (b).

- Real-time avoidance experiment

In this system, we added feedback information of surgical instrument when it was inserted into the organ. The color of surgical instrument's 3-D image changed automatically from green to red when the tip point of instrument approach critical area as shown in Fig.12 (a). This purpose of experiment was to examine the accuracy of this navigation system by avoiding a set of poles with different diameters. The processes of avoidance experiment are performed as follows. First, we inserted the surgical instrument toward the 3-D image of pole until the IP image's color of tip point change. When the color turn to red, we considered the surgical instrument approached the critical area. In order to confirm our imagination, we inserted the instrument about $2 \mathrm{~mm}$ at the same direction. If we perceived the instrument touching the pole, the experiment was recognized success. Conversely, if the touch could not be perceived or the color did not change through the experiment, the avoidance was judged as failure.

We conduced procedures four times for both of shallow location and deep location for phantom $\square$ and phantom $\square$ respectively. We succeed in avoiding the pole bigger than $3 \mathrm{~mm}$ both in shallow and deep location for 6 times, and succeed avoiding the $2 \mathrm{~mm}$ 's pole in shallow location but fail in deep location. After avoiding critical area, we continue to insert the surgical instrument, as shown in Fig.12 (b). 
(a)

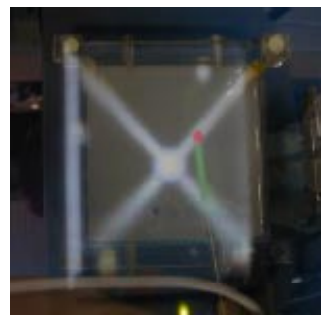

(b)

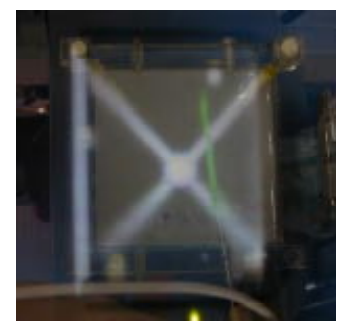

Fig.12 The color on the tip of instrument change to red when the instrument approaching critical area (a). Continue to stick after avoiding critical area (b).

\section{Discussion and Conclusions}

The navigation system developed in this study is more accurate by using a real-time 3$\mathrm{D}$ guided-image for intra-operative surgical instrument. To minimal invasion surgery, we developed an image-guided surgical system with 2 primary merits by IP.

- It is not necessary for surgeon to look away from the patient to receive guidance. Moreover, it avoid the need of extra devices, such as the wearing of special glasses;

- Geometrical accuracy over the projected objects (esp. depth perspective), visibility of motion parallax over a wide area, simultaneous observation by many people.

Based on the requirements above, we created organ's IP and offer a guidance for surgical instrument by showing the intra-operative location of surgical instrument which cannot be seen by observer when it enter the inside of organ respectively. Because soft tissues may deform during surgery, a method to sense intra-operative organ deformation and to display accurately their shapes and positions will be required. Although the update of organ's IP still costs time and the IP image is not very clear due to the limitation of pixel density of the presently available display device, with the advancement of display technology and computer hardware, it will be possible to register the intra-operative organ's configuration with the surgical instrument in real-time based on the real-time data of organ from Open-MRI or deformation calculation.

The resolution of flat display like LCD in this system is limited to 200 dpi. The spatial resolution of the 3-D image projected is proportional to the ratio of the lens diameter in the lens array to the pixel pitch of display. Thus, both the lens diameter and the pixel pitch need to be made much smaller. One of the possible solutions to realize higher pixel density is the use of multiple projectors to create one image and reduction projection of the resultant image on a small screen with special lens optics. A large computation power is also needed to correspond to multiple-projector system. We are currently developing such a multiple projector system and a parallelcalculation method to accelerate calculation.

In conclusion, our research has shown that the real-time 3-D surgical navigation system can superimpose the real, intuitive 3-D image for medical diagnosis and operation. The newly developed real-time IV algorithm for calculating the 3-D image 
of surgical instruments was effective to present the real-time location of surgical instruments and the organ during the operation. The experimental results show the errors of this navigation system were in the range of $2 \sim 3 \mathrm{~mm}$. Because of the simplicity and the accuracy of real-time projected point location by introducing a display device with higher pixel density, this system will be practically usable in the medical field.

\section{Acknowledgements}

This studies was partly supported by the research of Future Program JSPS-RFTF 96P00801 by Japan Society for the Promotion of Science.

\section{References}

1) M.Blackwell, C.Nikou, A.M.Digioia, T.Kanade, "An Image Overlay System for Medical Data Visualization," Mediacal Image Computing and Computer-Assisted Intervention MICCAI'98, pp.232-240

2) M.G.Lippmann, "Epreuves reversibles donnant la sensation du relief”, J. de Phys Vol.7, 4th series, pp821-825, 1908

3) Y.Masutani, M.Iwahara, etc al "Development of integral photography-based enhanced reality visualization system for surgical support. Proc. of ISCAS'95 pp16-17

4) Y.Igarashi, H.Mutrata, M.Ueda, "3-D display system using a computer generated integral photography. Japan J. Appl. Phys. 17 (1978) pp.1683-1684

5) S.Nakajima, K.Masamune, I.Sakuma, T.Dohi, "Three-dimensional display system for medical imaging with compute-generated integral photography", Proc. of SPIE Vol. 3957 (2000) pp.60-67

6) S.Nakajima, S.Orita, K.Masamune, I.Sakuma, T.Dohi, and K.Nakamura "Surgical Navigation System with Intuitive Three-Dimension Display", Mediacal Image Computing and Computer-Assisted Intervention MICCAI 2000, pp.403-411 